\title{
Towards quantitative elemental mapping across interfaces by combining momentum-resolved STEM and EDX
}

Mauricio Cattaneo ${ }^{1}$, Katherine MacArthur ${ }^{2}$, Juri Barthel ${ }^{1}$ and Knut Müller-Caspary ${ }^{1}$

${ }^{1}$ Ernst Ruska Centre for Microscopy and Spectroscopy with Electrons and the Peter Grünberg Institute Forschungszentrum Jülich, 52425 Jülich, Germany, United States, ${ }^{2}$ Ernst Ruska Centre for Microscopy and Spectroscopy with Electrons and the Peter Grünberg Institute Forschungszentrum Jülich, 52425 Jülich, Germany, Germany

In recent years, two branches of electron microscopy have seen major progresses in instrumentation. The introduction of fast cameras to scanning transmission electron microscopy (STEM) has given rise to momentum-resolved STEM where full diffraction patterns are recorded for each scan position. Real and reciprocal space information is thus simultaneously available in a four-dimensional dataset (4D STEM). In energy dispersive X-ray (EDX) spectroscopy, there is a combined improvement from silicon drift detectors, increasing detection efficiency, and aberration correction, increasing the available current density. The resulting higher counts in EDX data allows for atomic-scale maps on beam resilient samples.

Two challenges currently prevent our ability to accurately quantify composition across interfaces: electron channelling which causes the EDX signal to depend on both composition and atomic ordering [1,2], and beam scattering which makes an interface appear more diffuse than it actually is [3]. Sample tilt has been proposed as a method for ameliorating channelling effects and improving quantification, but the effects of beam broadening persist. By forward modelling, using simulations in direct comparison to experimental data, it is possible to separate structure information including chemical composition from beambroadening effects. However, this approach requires accurate sample information as input to simulations, including atomic structure, sample tilt, and thickness. Since these quantities potentially change across interfaces, we propose to perform momentum-resolved STEM in combination with EDX elemental mapping, i.e. to acquire a 5D STEM data set, with the aim to extract structural and compositional information.

5D STEM of a DyScO3-SrTiO3 (DSO-STO) multilayer sample is demonstrated with a probe-corrected Thermo Fisher Titan G2 electron microscope operated at an accelerating voltage of $200 \mathrm{kV}$. The microscope is equipped with a four quadrant Super-X EDX detector for elemental mapping and an electron microscope pixel array detector (EMPAD) for recording diffraction patterns at each scan position. An HAADF STEM image of the multilayer is shown in Fig. 1 together with a colour composition of elemental maps, both revealing atomic layer contrast under the large sample tilt which was intentionally applied to suppress channelling effects. Position averaged convergent beam electron diffraction patterns (PACBEDs) as shown in Fig. 2 were produced from the scanned diffraction data by averaging patterns from the centres of each DSO and STO layer. From these patterns, an average sample tilt of $66 \pm 1 \mathrm{mrad}$ along the interface and $2 \pm 1 \mathrm{mrad}$ perpendicular to the interface was measured, and 
no significant variation of tilt between layers was found. A sample thickness of about $20 \mathrm{~nm}$ was estimated by comparing the bright features of the PACBEDs to simulations. In a first attempt to apply these additional parameters in an EDX STEM simulation [4] for an abrupt interface, see inset Fig. 1 (b), clearly less delocalization of EDX signal is expected compared to the experiment. In conclusion, some degree of intermixing at the interfaces needs to be considered in the structure model.

(a)

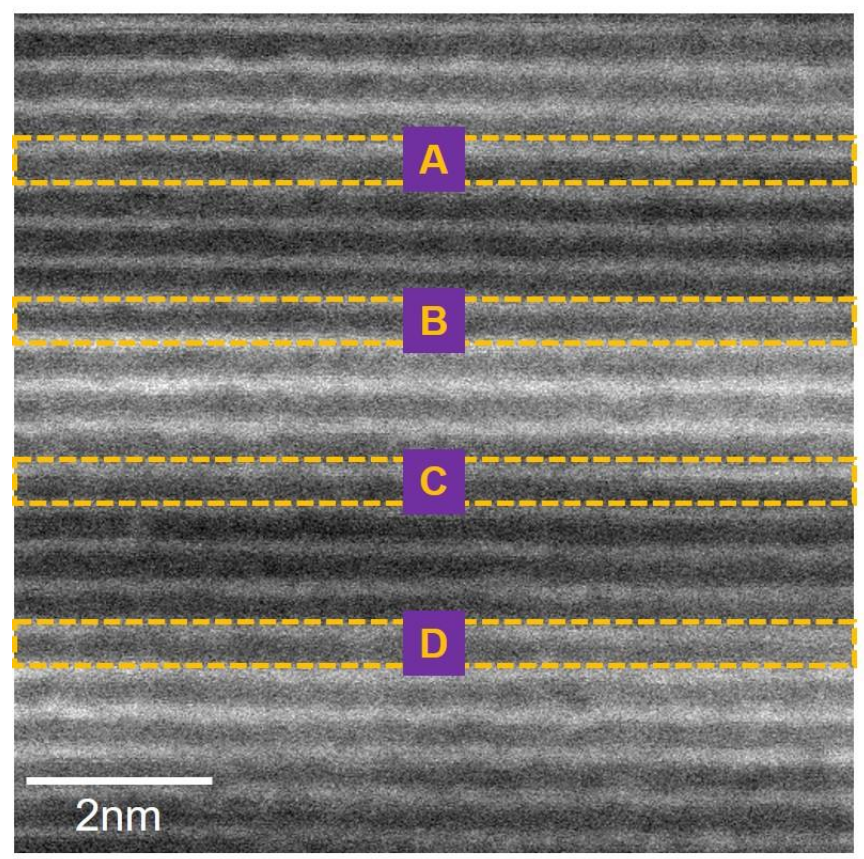

(b)

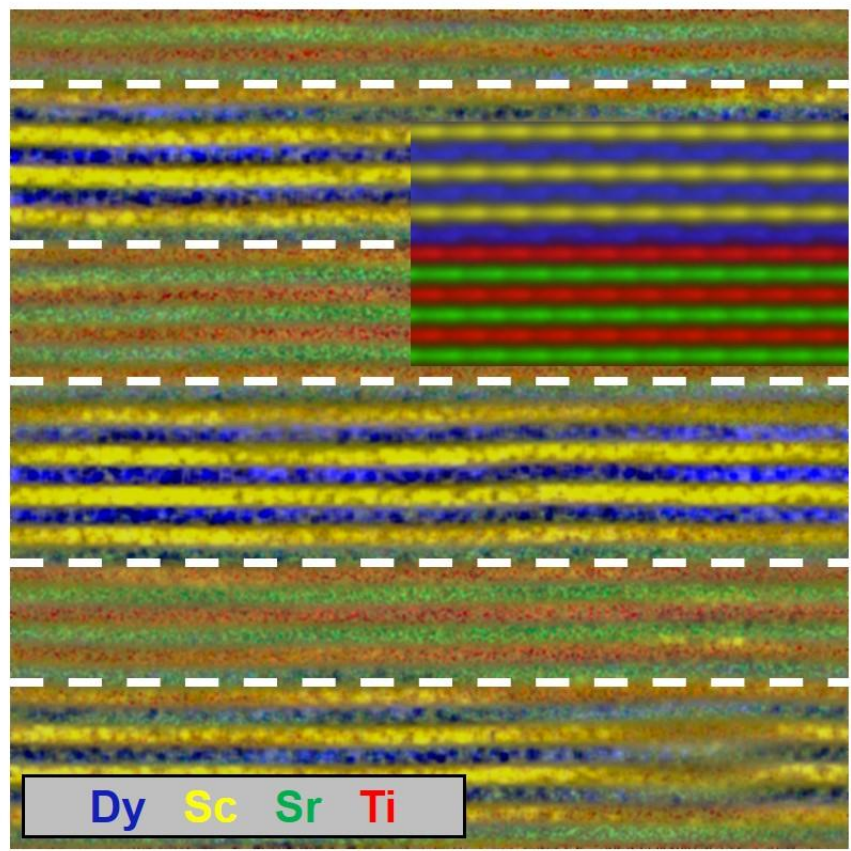

Figure 1. (a) STEM HAADF image and (b) elemental mapping of a DSO-STO multilayer sample under large sample tilt parallel to the layers. PACBED patterns shown in Fig. 2 were generated from the areas marked in (a). Atomic plane contrast in the composed elemental map has been enhanced by noise filtering for demonstration purpose. Dashed horizontal lines mark the position of interfaces. A respective elemental composition map from simulations for an atomically sharp interface, assuming tilt and thickness as estimated from the PACBEDs in Fig. 2 is shown as inset. 
(a)

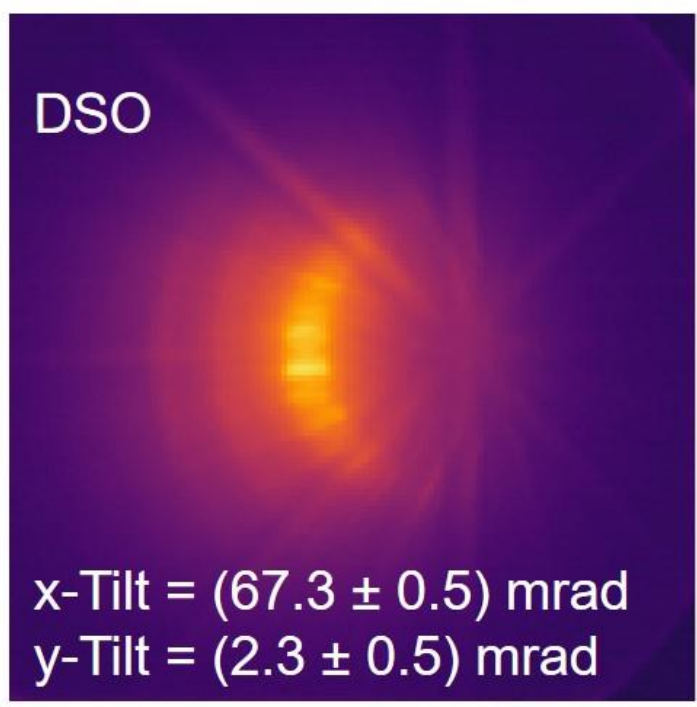

(c)

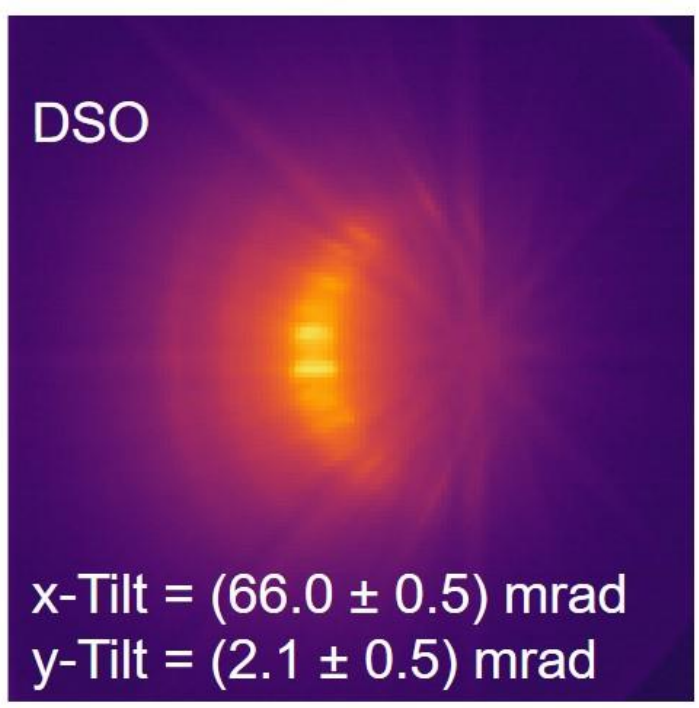

(b)

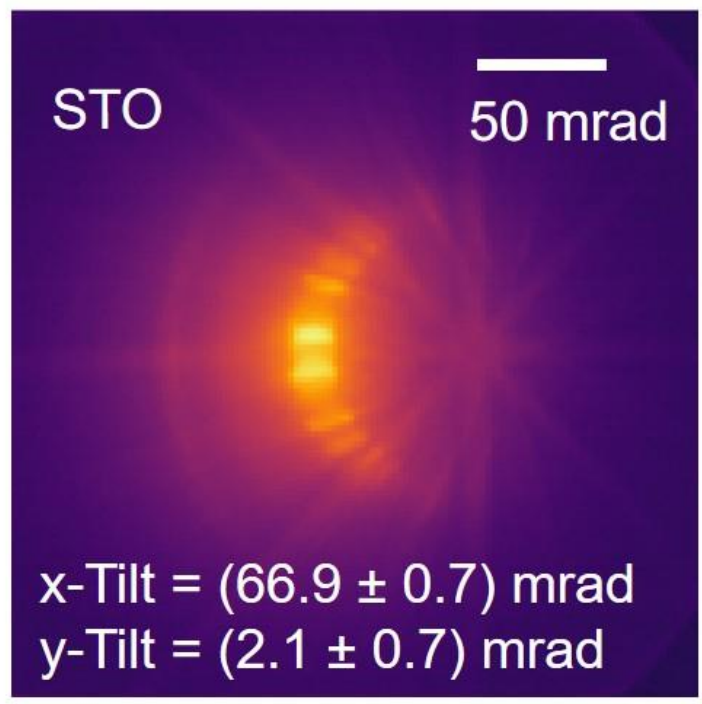

(d)

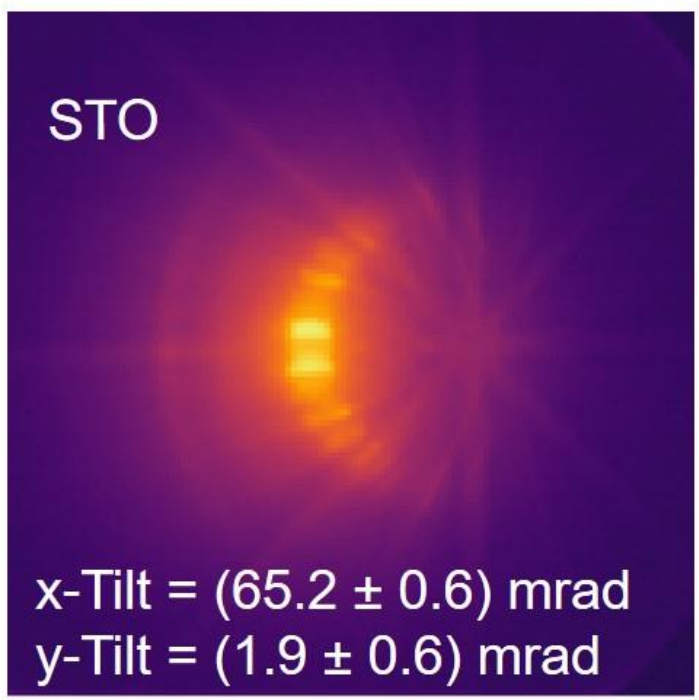

Figure 2. Diffraction patterns, averaged from the centre of $(a, c)$ DSO layers and $(b, d)$ STO layers as marked in Fig. 1a. No significant change of sample tilt is found between the four regions. The bright parts of the patterns also show no strong difference between layers of the same structure, indicating constant sample thickness in this case.

References

[1] N.R. Lugg et al., Ultramic. 151 (2015)

[2] K.E. MacArthur et al., Ultramic. 182 (2017)

[3] S.R. Spurgeon et al., Microsc. \& Microanal. 23 (2017) 
[4] L.J. Allen et al., Ultramic. 151 (2015)

[5] Funding: Jülich Melbourne Postgraduate Academy (JUMPA, M.C.), DFG HE 7192/1-2 (K. M.). and Helmholtz Contract No. VH-NG-1317 (K. M-C.) 